\title{
Introducción del enfoque de género en las carreras de la Universidad de La Habana. El caso de Bioquímica y Biología Molecular, Psicología y Sociología
}

Introduction to gender approach in the majors at Habana University. The case of Biochemistry and Molecular Biology; Psychology and Sociology

\section{ISSN 2071-8748 \\ E-ISSN 2218-3345 \\ (c) (1) $\$$ \\ BY NC SA}

Tania Caram-León Mayra Tejuca-Martínez

Niuva Ávila-Vargas

Danay Díaz-Pérez

Geydis Fundora-Nevot

Recibido: $24 / 07 / 18$ Aprobado: 12/12/18

URI: http://hdl.handle.net/11298/898

DOI: http://dx.doi.org/10.5377/entorno.v0i66.6736

\section{Resumen}

En la época de desigualdades en que vivimos, una de las más significativas es la de género. La importancia de su inclusión en los diferentes niveles de educación, y específicamente en las carreras universitarias, ha sido tratada en numerosos estudios dentro y fuera del país. En esta investigación pretendemos analizar comparativamente el proceso de introducción de la perspectiva de género en las carreras Bioquímica y Biología Molecular, Psicología y Sociología, atendiendo a

\section{Abstract}

In this epoch of inequalities in which we live, one of the most relevant is gender. The importance of its inclusion in the different educational levels, specifically in university careers, has been the object of numerous studies within and outside this country. Our purpose in this investigation is to establish a comparative analysis of the introductory process in the majors: Biochemistry and Molecular Biology, and Psychology and Sociology as per the study programs D and E at the La Habana

1 Las profesoras Tania Caram León (tcaram@flacso.uh.cu; Código ORCID: 0000-0001-7096-2254), Danay Díaz Pérez(danay@flacso.uh.cu; Código ORCID: 00000002-9988-4314) y Geydis Fundora Nevot (geydis@flacso.uh.cu; Código ORCID: 0000-0001-8450-9936)pertenecen a la Facultad Latinoamericana de Ciencias Sociales (FLACSO-Cuba); la profesora Mayra Tejuca Martínez (mayra@ceed.uh.cu; Código ORCID: 0000-0002-0147-3737), al Centro de Estudio de la Economía Cubana (CEEC) y Niuva Ávila Vargas (niuva@ffh.uh.cu; Código ORCID: 0000-0002-1992-7936), al Departamento de Sociología, pertenecientes a la Universidad de La Habana. 
Ios planes de estudio D y E en la Universidad de La Habana (UH) mediante análisis de documentos y entrevistas a expertos.

\section{Palabras clave}

Educación superior; Género; Currículo; Universidad de La Habana.
University (HU) by means of documental analysis and experts' interviews.

\section{Keywords}

Higher education, Gender, Curriculum, La Habana University.

\section{Introducción}

De acuerdo con Karl Mannheim, el proceso educativo moldea a las personas en una determinada colectividad, que debe ajustarse a los modelos predominantes de acción, y que se insertan en un orden social. Los fines educativos y su contexto social se transmiten a la nueva generación junto con las técnicas educativas dominantes (Albornoz, 1981). Se destaca así el papel que desempeña la educación como factor socializador y vehículo transmisor de la cultura. Así mismo, el pedagogo Denomme se refiere a la tríada que forman los agentes: estudiantes, enseñanza y medio ambiente, enfatizando que merecen mucha atención porque constituyen una cierta manera de interconexión de pedagogía interactiva. Según este autor los estudiantes y la enseñanza no son seres abstractos, ellos son el continuo actuar de un mundo físico, social y cultural que posee su propia identidad (Denomme y Roy, 1998). Para Nelly Stromquist (1998) la educación se convierte en el instrumento esencial de transformación que implica el proceso de empoderamiento.

La sociología de la educación, según Perrenoud (1997) no cesa de mostrar evidencias sobre la distancia entre la cultura, de una parte y las diferencias específicas que imprimen las clases sociales, las familias y sus culturas específicas. Estas discrepancias exigen un carácter distintivo a la educación, que tiendan a disminuir las desigualdades que podrían existir en el contenido educativo. Este enfoque resulta revelador por la potencialidad de su significado como mecanismo de acción y proyección social futura, partiendo de cambios en las formas de comprensión y de la asimilación de conocimientos.

Las instituciones educativas son responsables, en conjunto con otros agentes de socialización, de formar saberes, capacidades, habilidades, valores, entre otros, que orienten a los sujetos a la producción y reproducción de la sociedad. En este sentido, su rol en la historia de la humanidad ha tenido un doble rasero: 1) Como instituciones de control (Foucault), aparatos ideológicos del Estado (Althusser) al servicio del poder dominante, 2) Como medio de promoción del cambio y la emancipación. Ellas han tenido una responsabilidad preponderante en la creación y mantenimiento del sistema patriarcal ${ }^{2}$, en la construcción del sexismo, de identidades de género binarias, de la visión androcéntrica de la historia, de la heteronormatividad. Por ende, en las mismas, se encuentra una de las claves fundamentales para deconstruir este sistema de dominación que atenta contra el desarrollo de sociedades justas, equilibradas, inclusivas y armónicas.

Mención especial merece la educación superior. Las universidades no solo tienen la misión de la enseñanza. En el caso cubano, son tres sus funciones sustantivas: la docencia, la investigación y la extensión universitaria. Esto significa que su contribución a la permanencia o ruptura con la organización patriarcal, también se ubica en la producción de nuevos conocimientos científicos y su aplicación a diferentes esferas de la vida económica, social, política y cultural. De ahí la pertinencia de aplicar la perspectiva de género para analizar las prácticas tradicionales en el ámbito universitario y planificar los cambios pertinentes, si

\footnotetext{
2 El patriarcado, según Celia Amorós, es un conjunto metaestable de pactos entre los hombres, por el cual se constituyen en colectivo de estos como génerosexo, y correlativamente el de las mujeres. El contenido de sus pactos son las mujeres. Esta desigualdad entre los sexos se mantiene porque los hombres cuentan con los medios políticos, económicos, ideológicos y físicos para que subsista, independientemente de lo que las mujeres puedan desear. En este sentido, la violencia forma parte del núcleo estructural del patriarcado y su proceso primordial de reproducción es la sexualización de las personas. (Amorós, 1990; p. 49. En: De León, C. Escuela de Formación Política Feminista. Módulo Genealogía feminista, 2010. Guatemala: La Otra Cooperativa/Editorial La Trilla).
} 
se pretenden sistemas educativos en pos del bienestar y desarrollo de toda la ciudadanía.

En la Agenda Mujeres publicada en Cuba (2017), espacio donde convergen saberes desde la academia, el activismo social y político, las redes, los medios de comunicación, etc.; la perspectiva de género se define como herramienta conceptual que permite analizar el impacto del género en las oportunidades, roles e interacciones sociales de las personas $y$ transversalizar programas, políticas $y$ organizaciones desde un enfoque de género. Cuestiona los estereotipos en que las personas son educadas desde la infancia y promueve la posibilidad de elaborar nuevos contenidos de socialización y relación.

Desde el diseño de los currículos y en el propio currículo oculto se expresan múltiples desigualdades, entre ellas las de género. En esta investigación se realizó un análisis comparativo sobre el proceso de introducción de la perspectiva de género en las carreras de Bioquímica y Biología Molecular, Psicología y Sociología atendiendo a los planes de estudio D y E en la Universidad de La Habana, a través de revisión bibliográfica y entrevista en profundidad.

\section{Metodología}

Para la realización de la investigación nos propusimos los siguientes postulados:

Problema de investigación:

- ¿Cómo ha sido el proceso de introducción de la perspectiva de género en las carreras Bioquímica y Biología Molecular, Psicología y Sociología atendiendo a los planes de estudio D y E en la Universidad de La Habana?

Objetivo general:

- Analizar comparativamente el proceso de introducción de la perspectiva de género en las carreras Bioquímica y Biología Molecular, Psicología y Sociología atendiendo a los planes de estudio $\mathrm{D}$ y $\mathrm{E}$ en la Universidad de La Habana.

Objetivos específicos:

- Analizar el proceso de introducción del tema de género en la Universidad de La Habana.
- Caracterizar los planes de estudio D y E en cuanto al tratamiento de género en las carreras escogidas.

- Valorar las modificaciones que tuvo el plan E respecto al anterior plan $D$ en materia de género, en las carreras estudiadas.

- Comparar la introducción del tema de género según carreras seleccionadas.

Para dar cumplimiento a los objetivos propuestos se realizó primero una revisión bibliográfica que contó con textos que reseñan precisamente la historia del tratamiento e introducción del tema de género en la Universidad y específicamente en las carreras de Bioquímica y Biología Molecular, Psicología y Sociología.

Los métodos y técnicas utilizadas fueron fundamentalmente dos: análisis de contenido a los planes de estudios $\mathrm{D}$ y $\mathrm{E}$ de las tres carreras seleccionadas y entrevistas a expertos. Estos últimos fueron todas mujeres pertenecientes a: la Dirección Docente Metodológica de la Universidad de La Habana, la presidenta de la Cátedra de la Mujer y una profesora del grupo de investigación: «Género, Sexualidad y Familia», también de la propia Universidad de La Habana.

\section{Variables e indicadores}

Las dos variables que se midieron fueron a) proceso de introducción de la perspectiva de género $y$, b) planes de estudio $D$ y E. Los indicadores que ayudaron a cumplir la primera medición fueron: Antecedentes de la introducción de la perspectiva de género en la Universidad de La Habana, Creación de la Cátedra de la Mujer, Incorporación del tema género en los planes de estudio y en las asignaturas electivas. Y para la segunda se tuvieron en cuenta: Características de los planes de estudio, sistema de conocimiento de las asignaturas, cantidad de asignaturas que abordan el tema de género y por tipo de currículo.

\section{Resultados y discusión}

\section{Características de los planes de estudio}

Para comprender cómo se ha introducido el enfoque de género en las carreras universitarias cubanas es importante conocer las características generales de los planes de estudio (MES, 2016).

El organismo rector de la enseñanza universitaria cubana es el Ministerio de Educación Superior (MES), creado en 1976. 
Una de sus funciones principales es el perfeccionamiento continuo de los planes de estudio con vistas a garantizar el sostenido incremento de la calidad en el proceso de formación. Desde 1977 hasta 2016 se han aplicado cuatro generaciones de planes de estudio, los que se denominaron de forma consecutiva A, B, C y D. En el curso 2015-2016 se dieron las indicaciones para la elaboración e implementación de nuevos planes de Estudio (E). Hoy estos nuevos planes están siendo aplicados en muchas carreras a lo largo del país.

En la Universidad de La Habana, en el recién terminado curso escolar 2017-2018, en 21 de 38 carreras se había ya comenzado a implementar los nuevos planes de estudio. Entre ellas se encontraba la carrera de Psicología, mientras que la carrera de Sociología y la de Bioquímica y Biología Molecular comenzarán su puesta en práctica en el próximo curso escolar.

Los planes de estudio E, así como la generación anterior (los D) se caracterizan por la flexibilidad curricular que se manifiesta, fundamentalmente, por la existencia de tres tipos de contenidos curriculares que dan lugar a los currículos base, propio y optativo/electivo. Esta estructura permite la actualización permanente del plan de estudio de las carreras y su adaptación a las necesidades del país, del territorio, al desarrollo del claustro y a los intereses de los estudiantes. Las características de los tres tipos de contenidos curriculares son:

- Contenidos curriculares de carácter nacional (currículo base): se determinan centralmente por las Comisiones Nacionales de Carrera y son de obligatorio cumplimiento para todas las universidades en que se desarrolla la carrera. Aseguran el dominio de los modos de actuación del profesional. A partir del currículo base, y teniendo en cuenta los objetivos generales de la carrera, cada centro decide el modo de completar su plan de estudio particular, en correspondencia con sus características y las de su territorio, a través de los currículos propio y optativo/electivo.

- Contenidos curriculares propios (currículo propio): se deciden por cada universidad. Incluye contenidos dirigidos a satisfacer necesidades específicas del desarrollo regional. Son de obligatorio cumplimiento para todos los estudiantes que cursan la carrera en dicho centro de educación superior.
- Contenidos curriculares optativos y electivos (currículo optativo/electivo): se ofertan por cada universidad, y son elegidos por los estudiantes. Sirven como complemento para su formación integral y dan respuesta a los intereses de desarrollo personal de cada estudiante. En la modalidad electiva pueden seleccionarse asignaturas que se imparten en otras carreras.

Por otro lado, uno de los desafíos de la educación superior cubana es lograr que la formación y desarrollo profesional se acompañe de responsabilidades ética, social y ambiental. Es decir, que el egresado no solo demuestre una alta calificación en su desempeño profesional, sino que posea cualidades personales que lo ayuden a conjugar sus intereses personales con los de la sociedad y participe activa, crítica y constructivamente en el desarrollo de esta.

Para lograr esto, en los contenidos propios de las carreras se integran estrategias curriculares. Estas estrategias se refieren a otros aspectos que brindan una formación más integral al estudiante en respuesta a exigencias científicotécnicas y sociales. Tales estrategias deben ser insertadas a lo largo de la carrera teniendo en cuenta siempre las potencialidades que ofrecen los contenidos que se explican en las disciplinas y asignaturas propias de la especialidad.

En virtud de ello, el documento base para el diseño de los planes de estudio «E» orienta prestarle especial atención al uso de la lengua materna; de las tecnologías de la información y las comunicaciones en la solución de tareas de aprendizaje; al uso del idioma inglés; a la realización de valoraciones económicas, ambientales, políticas, jurídicas y sociales; y a la inclusión, de forma coherente, de aspectos relacionados con la preparación para la defensa del país.

En cualquier caso, las estrategias curriculares son un componente flexible, en el sentido de que en cada carrera se instrumentan de manera particular y que, además, pueden ser enriquecidas con otras estrategias que las comisiones de carrera consideren importantes para las especialidades que dirigen.

\section{Antecedentes}

En Cuba el programa de Cátedras de la Mujer, creado y coordinado por la Federación de Mujeres Cubanas en 1989, tiene entre sus objetivos contribuir a borrar prejuicios y estereotipos desde la educación, así como formar el valor 
de la igualdad de oportunidades, posibilidades y derechos entre mujeres y hombres (CEM, 2012). Es por ello que las cátedras han desarrollado diferentes estrategias para lograr la inserción del enfoque de género en la docencia universitaria. En la Universidad de La Habana la cátedra se fundó en 1991.

El trabajo de las cátedras es atendido desde 1997 por el Centro de Estudios de la Mujer (CEM), que organiza talleres nacionales con cierta periodicidad para darle seguimiento a su cometido. Se destaca entre estos eventos el IX Taller Nacional de Cátedras de la Mujer "

«Buenas prácticas en la introducción del enfoque de género en la Educación Superior», celebrado en octubre de 2012 (CEM, 2012).

En el mencionado taller, y con la presencia del ministro de Educación Superior, se llegó a consenso acerca de la importancia de incluir el enfoque de género en las estrategias curriculares de los planes de estudio universitarios. No obstante, en las estrategias curriculares propuestas en el documento base para el diseño de los planes de estudio «E» no se incluyó esta perspectiva (MES, 2016).

Sin embargo, durante la revisión de las disciplinas de formación general, comunes a todas las carreras, encontramos en las disciplinas Marxismo-Leninismo, e Historia de Cuba alusión a estos temas. En la primera de ellas, la lucha contra todas las formas de discriminación: racial, de género, étnica, territorial y otras, aparece entre los conocimientos esenciales por adquirir. Igualmente se menciona el enfoque de género entre las estrategias curriculares de la disciplina ${ }^{3}$. En el caso de la disciplina Historia de Cuba aparece en sus documentos normativos la orientación de abordar los problemas de género que existían en el país en la etapa republicana, anterior al triunfo revolucionario.

De manera que, a través de estas disciplinas de formación general debe abordarse el enfoque de género en todas las carreras universitarias. Queda pendiente un análisis sobre cómo se materializan estas indicaciones en la práctica y si son suficientes estas aproximaciones al tema para logar una conciencia de género en el estudiantado en carreras distantes de estas temáticas.

Por lo tanto, la decisión de incluir el enfoque de género en las disciplinas propias de las distintas especialidades corresponde a las comisiones de carrera. Es de esperar que solo aquellas en las que se imparten asignaturas directamente relacionadas con estos temas esté presente el enfoque de género.

\section{Tratamiento del tema género en las carreras estudiadas}

\section{Carrera de Bioquímica y Biología Molecular}

Para este estudio se escogió la carrera de Bioquímica y Biología Molecular como representante del grupo de las Ciencias Naturales y Matemática de la Universidad de La Habana. Como anticipábamos, el enfoque de género solamente está presente en el plan de estudios E de la carrera en las disciplinas Marxismo-Leninismo e Historia de Cuba (MES, 2017). Como ya se había comentado, ambas son disciplinas de formación general, comunes a todas las carreras. Por tanto, podría decirse que más allá de lo que estas asignaturas puedan brindar en el tema de género, en esta carrera no existe un propósito, al menos declarado, de introducir el enfoque de género en la formación profesional.

\section{Carrera de Psicología}

Una de las carreras donde más se abordan las cuestiones de género es la de Psicología. En el plan D de esta se manifiesta que el profesional formado debe estar comprometido con los estudios de cuestiones sociales de interés estatal, entre ellos el cambio de la participación social de la mujer. De igual forma deberá «dar explicaciones sobre los procesos de cambios que ocurren en el ser humano durante su desarrollo, en el aprendizaje y en la paulatina inserción en espacios sociales diferenciados, desde la infancia hasta la vejez, teniendo en cuenta su diversidad cultural, lingüística, racial, de género (...)» (MES, 2009).

Mediante el análisis de contenido realizado al plan D de la carrera de Psicología se pudo comprobar que el género se aborda en disciplinas como: Psicología Social (asignatura

\footnotetext{
3 En algunas universidades cubanas como en la Agraria de La Habana Fructuoso Rodríguez, la Cátedra de la Mujer radica justamente en el departamento de Marxismo-Leninismo. En este caso la asignatura Filosofía se ocupa de tratar los temas de género (CEM, 2012).
} 
Desviación de la conducta), Psicología de la Personalidad (asignaturas Psicología de la Personalidad I y II), Psicología del Desarrollo Humano (asignatura Psicología del desarrollo de las edades tempranas y preescolares), Psicología Clínica y de la Salud (asignatura Orientación y terapia sexual y de pareja), Prácticas de producción en Psicología (Psicología de la Familia) y Marxismo-Leninismo. Además, dentro de las indicaciones metodológicas y de organización de la carrera de Psicología se recomienda considerar a partir de tercer año asignaturas como la Psicología del Género y, dentro de las opcionales que se ofrecen en quinto año, asignaturas como Género, subjetividad y vínculo amoroso.

Actualmente la carrera de Psicología, con la participación de sus estudiantes, realiza un experimento metodológico en el diseño curricular por competencias que se plasmará en el Plan de Estudios E. Una de las acciones será la revisión de las disciplinas para aproximar sus objetivos a las competencias definidas y continuar perfeccionando el proceso de enseñanza-aprendizaje (Facultad de Psicología, 2018). Según el nuevo Plan de Estudios E las estrategias curriculares deben integrarse coherentemente como objetivos y saberes profesionales de carácter interdisciplinario y que responde a: formación ideológica, lengua materna, educación para la salud, la sexualidad responsable, el enfoque de género y racial, educación jurídica y medioambiental, desarrollo de la creatividad, orientación profesional, cultura económica y tributaria y otros (MES, 2017).

Desde la investigación también se promueven estudios de género tanto en el pregrado como en el posgrado. Es meritorio destacar que la Facultad de Psicología tiene una Maestría en Estudios de Género donde se han desarrollado investigaciones sobre la violencia contra la mujer, la maternidad adolescente, el rol de la paternidad posdivorcio, identidad de género, entre otros. Resulta de especial interés la Tesis de Maestría de Escalona Peña (2012) «Fundamentación de una propuesta para incluir los estudios de género en la formación curricular de la carrera de Periodismo». El estudio presenta una ausencia del enfoque de género en la formación profesional de periodistas y su repercusión en los productos comunicativos y la construcción de noticias; por lo que la autora propone su incorporación tanto en la docencia como en la investigación.

\section{Carrera de Sociología}

Para poder entender la magnitud de lo logrado por el claustro de Sociología, habría que comenzar diciendo que es esta la primera carrera en el país que ubicó una asignatura relacionada con los estudios de género en el currículum propio de la especialidad. A partir de la reapertura en el año 1990, el Departamento de Sociología tuvo entre sus prioridades el desarrollar investigaciones sobre las mujeres, lo que fue favorecido con la conformación de un Grupo de Investigación sobre género, sexualidad y familia, solo un año después.

Sin lugar a dudas este hecho y toda la producción y actividad científica asociada a él, ha generado un ambiente propicio para la educación de los estudiantes en temáticas de género. Ello no solo se traduce en conocimientos prácticos o teóricos aprendidos como parte del currículo por vencer para su licenciatura, sino que estos se han apropiado también de estas temáticas y la han convertido en sus temas de investigación. En el «Informe para la acreditación de la carrera» de Sociología realizado en 2012 se confirma que el $28 \%$ de los diplomas defendidos durante 2007 y 2012 se refieren a temas de género (Romero, Proveyer y Fleitas, 2017); y otro porcentaje, también relevante, incorpora al menos un objetivo específico sobre género dentro de sus investigaciones de diploma.

El proceso de incorporación como parte de la docencia en la carrera se empieza en 1994, teniendo como antecedente todo el quehacer del recién creado grupo, antes mencionado. Los resultados de trabajos, intercambios académicos, estudios y superación de las profesoras del departamento hicieron posible que los estudiantes de cuarto año comenzaran a contar con una asignatura optativa. Por lo que se pudiera decir que ya desde los primeros graduados y la reapertura de la carrera, se pensó en formar un profesional capacitado en temas de género. Y, si bien solo era una asignatura optativa de 20 horas, que impartía la profesora Dra. Clotilde Proveyer, su estabilidad y sistematicidad hizo que posteriormente se lograra incluir como asignatura obligatoria (Romero, Proveyer y Fleitas, 2017: 229).

Para el año 1998, con solo cuatro años de experiencia dentro del currículo de la carrera se abre como asignatura obligatoria 
para aquellos graduados de la Licenciatura en Sociología con salida en Trabajo Social, durante el Plan de Estudios C. Los años sucesivos fueron aprovechados para crear solidez en un programa que luego de una década se incorporaría a los cursos regulares diurnos dentro del currículo propio de la especialidad para todas las modalidades. Lo más importante es que se sumarían las universidades de Las villas y de Oriente, extendiendo más aún las experiencias acumuladas por el claustro de la asignatura y del grupo de investigación. Todo ello sucedió durante la aplicación del Plan de Estudios
D, el cual no solo tiene como ganancia esta asignatura, sino la consolidación e incorporación de otras que presenten en sus sistemas de conocimientos o habilidades herramientas teóricometodológicas para el trabajo con perspectiva de género.

Como se muestra a continuación, durante este plan de estudios al menos cinco asignaturas incorporaban el tema de género en su sistema de conocimientos, de una forma u otra era evidente la necesidad y la prioridad que tenía para la formación profesional el enfoque de género.

Tabla 1. Asignaturas del Currículo Propio y Base que abordan el tema de género, según plan de Estudio.

\begin{tabular}{|c|c|}
\hline PLAN D (Sistema de conocimientos) & PLAN E (Sistema de conocimientos) \\
\hline $\begin{array}{l}\text {-Sociología política: La participación política desde una } \\
\text { perspectiva de género. } \\
\text {-Sociología del trabajo: Género y Trabajo: Principales enfoques } \\
\text { analíticos. } \\
\text {-Sociología de la salud: Las desigualdades de clase, raza, } \\
\text { étnicas, territoriales y de género y su relación con la enfermedad. } \\
\text { Género y salud. } \\
\text {-Sociología de la familia. } \\
\text {-Sociología de género. }\end{array}$ & $\begin{array}{l}\text {-Sociología de la Educación: Construcción social de la } \\
\text { diferencia en la vida escolar a partir de la clase, el género y la } \\
\text { etnia. } \\
\text {-Antropología Social: Tributa con sus conocimientos a temas de } \\
\text { actualidad que son de interés sociológico como el género } \\
\text {-Problemas de la Estructura Social y las Desigualdades: Los } \\
\text { ejes transversales de la ES y las desigualdades: género, razal } \\
\text { etnia, territorio y las categorías ocupacionales. }\end{array}$ \\
\hline
\end{tabular}

Al igual que la Sociología de Género, Familia dedica más de la mitad de los temas que aborda, al estudio de la problemática de género: desigualdades entre hombres y mujeres en el ámbito familiar. Pero además las habilidades que desarrolla en los estudiantes en su totalidad están pensadas para desarrollar el enfoque de género necesarios para un sociólogo.

Como muestra la Tabla 1, en el Plan de Estudios E que entrará en vigor el próximo septiembre de 2018, se incorporaron a los sistemas de conocimientos de tres asignaturas, temas asociados al género. Sin embargo, siguen existiendo otras que son potencialmente susceptibles para introducir explícitamente la temática: Sociología de la Comunicación; de la Cultura; de la Prevención Social; y de la Infancia.

Como parte importante de la labor que se realiza por los profesores del departamento y graduados que laboran en otros centros de investigación, y que son responsables de las asignaturas optativas, se puede señalar que, en ambos planes de estudio, cerca de la mitad de estas optativas tocan temas asociados al género:

- Panorama sociodemográfico cubano actual y perspectivo.

- Relaciones raciales desde una perspectiva de género, en la Cuba contemporánea.

- Masculinidad hegemónica y paternidades emergentes.

- Sociología del Turismo.

- Sociología jurídica.

- Sociología y política social.

Se ha intentado visibilizar la importancia del enfoque de género tanto para los análisis que se realizan en las aulas por profesores y estudiantes, como para motivar a estos últimos en su quehacer científico. La posibilidad que brindan las asignaturas, así como la exigencia de la realidad social en la que se insertan los estudiantes a través de investigaciones desde su primer año, son potencialidades que han sido aprovechadas por los profesores y estudiantes. 


\section{Conclusiones}

Durante la investigación se constató que solo se ha impartido un curso electivo sobre el tema de género por la Facultad Latinoamericana de Ciencias Sociales (Flacso), Programa Cuba, titulado: Mujeres en la Cuba actual: un balance entre espacios ganados y permanencia de retos; que algunas carreras han introducido el enfoque de género en su currículo y se diferencian por el grado que esto ocurre; que en el Plan E se pretende fortalecer el enfoque de género en aquellas carreras que ya lo tienen incorporado (Sociología y Psicología) y promoverlo en las que no lo han desarrollado.

Podemos concluir que la introducción de la perspectiva de género en la Universidad de La Habana permitiría que los estudiantes egresados se graduaran mejor preparados para su posterior desempeño profesional y personal, como se ha evidenciado en las carreras de Psicología y Sociología.

La inclusión de los temas de género en todos los niveles de educación, y particularmente en la Educación Superior, podría contribuir a la transformación de sociedades más justas y equitativas, pues son las nuevas generaciones con otros parámetros de comprensión, las que alcanzarán valores diferentes para aproximarse a una nueva forma de vivir, pensar, sentir y actuar.

\section{Recomendaciones}

Introducir más asignaturas electivas relacionadas con el tema de género que permitan la formación de profesionales más preparados.

- Incluir el enfoque de género en las estrategias curriculares.

- Promover el tema de género en las asignaturas del currículo base, ejemplo Historia de Cuba o Filosofía.

- Aprovechar las potencialidades que brinda poseer una Cátedra de la Mujer para la introducción -y evaluación posterior- del enfoque de género en la formación curricular del graduado universitario.

- Realizar talleres de capacitación en temas de género a los presidentes de las comisiones de carreras y extenderlo a todo el personal involucrado.

\section{Referencias}

Agenda Mujeres (2017): Colectivo de autores. Naciones Unidas en Cuba. La Habana.

Albornoz, Orlando (1981): Sociología de la Educación, $4 a$. Ed. Ampliada. Ediciones de la Biblioteca, Universidad Central de Venezuela, Caracas.

CEM (2012): Buenas prácticas en la introducción del enfoque de género en la Educación Superior. Memorias del Taller Nacional de Cátedras de la Mujer. Centro de Estudios de la Mujer. La Habana, Cuba.

De León, C. (2010): Escuela de Formación Política Feminista. Módulo Genealogía feminista. La Otra Cooperativa/ Editorial La Trilla. Guatemala.

Denomme, Jean Marc, y Madelaine Roy (1998): Por una Pedagogía interactiva, Gaetan Morin, Montreal.

Facultad de Psicología (2018): «Experimento metodológico: Diseño curricular por competencias» Plan de Estudio E. Universidad de La Habana.

MES. (2016): Documento base para el diseño de los planes de estudio «E». Ministerio de Educación Superior. Cuba.

MES. (2017): Plan de Estudios «E». Carrera Bioquímica y Biología Molecular. Ministerio de Educación Superior. Cuba.

MES. (2009): Plan de Estudios «D». Carrera Psicología. Ministerio de Educación superior. Cuba.

MES. (2017): Plan de Estudios «E». Carrera Psicología. Ministerio de Educación superior.

MES. (2008): Plan de Estudios «D». Carrera Sociología. Ministerio de Educación superior. Cuba.

MES. (2017): Plan de Estudios «E». Carrera Sociología. Ministerio de Educación superior.

Perrenoud, Philippe (1997): Pedagogía diferenciada: de las intenciones a la acción, E. S. F., París.

Romero Almodóvar, Magela; Proveyer Cervantes, Clotilde y Reina Fleitas Ruiz (2017): Algunas reflexiones sobre la transversalización del enfoque de género en la Educación Superior: la experiencia de la carrera de Sociología en la Universidad de La Habana. En: Romero Almodóvar, Magela y Clotilde Proveyer Cervantes. Género y Sociedad. Encrucijadas teóricas y alternativas para el cambio. Editorial Universitaria Félix Varela. La Habana, Cuba.

Stromquist, Nelly (1998): La búsqueda del empoderamiento: en qué puede contribuir el campo de la educación. En: Magdalena León, Poder y empoderamiento de mujeres, UN, Facultad de Ciencias Humanas, T/M Editores, Santa Fe de Bogotá. 